\title{
Remote Monitoring System of an Agricultural Tillage Machine Based on an Embedded ARM Technology Wireless Sensor
}

\author{
http://dx.doi.org/10.3991/ijoe.v12i05.5732 \\ Jiaqi He and Yong Chen \\ Sichuan Agricultural University, Sichuan, China
}

\begin{abstract}
To solve the problems of monitoring system topology structure and joint ductility, as well as to improve the efficiency of agricultural planting, this study presents a greenhouse-monitoring system based on the advanced RISC machine (ARM) wireless sensor network. The design schemes of the hardware and software of the network nodes are presented, and the process of a ZigBee network coordinator is elucidated. The system uses ARM to control the wireless transceiver module for data transmission and reception as well to collect data through an inter-integrated circuit bus. The working-state data of the seeding machine are transmitted to the monitoring platform through the ZigBee network, which can achieve real-time data view. Test results show that the system has the advantages of a simple structure, a flexible node, and low power consumption. The effective monitoring of the agricultural seeding machine in a wireless environment is realized. This system can quickly monitor the agricultural seeding machine while working, and the data center allows reading the data for remote viewing and analysis. This system can also effectively reduce the workload of farmers, improve the efficiency of field sowing, and promote the rapid development of modern agriculture.
\end{abstract}

Index Terms-Embedded, Remote monitoring of seeding machine, WSN

\section{INTRODUCTION}

With the rapid development of the information age, traditional farming has been replaced with information, and field work is also moving toward the information and mechanical age. The core idea of "precision agriculture" is to realize the sustainable development of agriculture according to the requirements of each field work element with minimal investment return. Wireless transmission technology remains within a small section of agricultural system research. By contrast, the industrial use of terminal technology for real-time transmission of remote data is relatively extensive. In the United States, Japan, and other agriculturally developed countries, this technology has gradually become mature and is widely adopted, including in electronic monitoring equipment and remote control devices. For example, a large agricultural seeding machine has a wide range of applications. Such technology is also widely used in field production and management for farmland precision seeding to improve the utilization of farmland resources, reduce the production cost of crops, enhance the quality of agricultural products and production, and decrease the damage to the environment. In 2015, two related sessions in China focused on the fields of agricul- ture, rural areas, and agricultural modernization. Agricultural modernization technology will be in the era of data information.

Most industrial applications are currently connected under a wired connection mode, which is used to accomplish and achieve various control functions. Wired networks have provided considerable convenience to the work and life activities of people and have played a role in promoting social progress. In practical applications, wired networks can provide a huge amount of data flow, speed, and reliability, as well as achieve desired results. With the development of wireless communication technology, the function of wireless communication has improved, the speed of wireless communication has accelerated, and the speed of cable transmission can be enhanced. Traditional wired network cabling is material- and time-consuming, difficult to detect, and not free to move. At present, people are accustomed to mobile and wireless communication. The value of wireless communication technology is particularly emphasized in cases where the working conditions of a wired network are poor. The applications of wireless communication technology are mainly manifested in underground coal mine gas monitoring, electric power communication, wireless equipment, and automobile instrument wireless data reading, among others. Subcarrier multiplexing is replaced with embedded products. Advanced RISC machine (ARM) conforms to current trends and continues to introduce new products to satisfy different requirements from all walks of life and to occupy a certain position. Over $75 \%$ of the market is presently occupied by ARM technology processor applications. Most smartphones and digital electronic products worldwide use ARM embedded processor systems. ARM processors exhibit good reliability and processing capability, and they can satisfy applications in many fields. The application of ARM processors is mainly embodied in electronic products, wireless communication, network applications, and industrial control, among others. Production and daily life cannot be separated from ARM processors. Therefore, ARM embedded technology and GPRS communication network can be utilized to establish a remote data transmission system in agricultural fields.

In other countries, many wireless data transmission systems are used in agriculture. Stanford University and the Massachusetts Institute of Technology jointly developed a remote monitoring and fault diagnosis technology for information exchange format and standards [1]. Agricultural wireless network and embedded technologies are also being rapidly developed in China. For example, Liu 
Jian et al. proposed a design scheme for a wireless environmental monitoring system based on GPRS in 2008 [2]. Guo Zhiwei et al. proposed an application layer communication protocol for identifying and transmitting farmland information collected through an enhancement system in 2009 , which reduced the incidence of information blocking [3]. Xia Ruxiao designed a remote temperature and humidity real-time monitoring system in 2012 [4]. According to the status quo both local and abroad, the main problems in existing remote wireless data transmission systems are system transmission efficiency, reliability, and safety problems.

The remainder of this paper is organized as follows. Section 2 discusses wireless sensor data fusion design. Section 3 provides the design of a college sports management decision-making system based on artificial intelligence. Section 4 presents the system experimental verification analysis. Section 5 concludes the study.

\section{STATE OF THE ART}

Commercial embedded systems have existed since the mid-20th century; however, most of these are proprietary systems. The development of the embedded system industry has entered the golden era. Many countries and enterprises are committed to research on this area.

The ARM Company mainly authorizes the technology; the company does not sell a specific chip, and only its design technology is for sale. At present, the ARM architecture processor has a strong competitive edge in wireless communication and embedded fields because of its high speed, low energy consumption, low cost, and other characteristics. Since the emergence of the first ARM prototype in the 1990s, the ARM architecture has been defined in six versions (from V1 structure to V6 structure). Most current ARM processors have V6 structure, which has an increased number of instructions, and thus, improved dataprocessing capabilities and performance.

The ARM9 series of the ARM processor architecture has large-scale applications in various industries compared with the classic series. The ARM9 series does not only support a management unit memory function but can also handle instructions and data. The ARM9 series of instructions on implementation efficiency is also fast (with five lines) and can satisfy the demands of wireless devices, handheld terminals, and other applications.

Wireless sensor network (WSN) technology is constantly improving. This technology has become a crucial research topic in various countries. With the development of the information technology (IT) industry, WSN technology is expected to gradually mature to perfection.

Most nodes in WSNs are powered by batteries, and battery life is limited. Therefore, we have to consider this problem in a WSN protocol. In such protocol, the media access control (MAC) and routing protocols consume the most power. These two protocols can be optimized to improve the usage time of the acquisition node. The task of the MAC protocol is to ensure that all nodes can use the wireless channel and will not affect one another. The main process of the routing protocol is to optimize the generation of such protocol, and a good routing protocol can reasonably control energy to prolong the lifetime of WSN.

Network security is a crucial aspect of WSNs. If the network transmission process is insecure, then it cannot be widely used. We need to ensure the security of WSNs and simultaneously guarantee that the communication process will be unaffected. We need to develop protocols that can deal with threats from a wide range of environments because WSNs are widely used and network threats vary.

A tree network is constructed using a coordinator connected with one or multiple star subnetworks. The network node has its own parent node or lower level nodes. Direct and internode communication must be accomplished by the routing nodes in the network.

A star network is the simplest network structure that forms the basis of other network topologies. This network is made up of a ZigBee coordinator and a plurality of terminal equipment. All communication in this network should follow the coordinator for forwarding.

Mesh networks are based on tree networks and can realize direct interconnection with routing nodes via routing tables. Therefore, network message delay can be considerably reduced, the reliability of the entire network is enhanced, and the storage space of the equipment is increased.

\section{MethodOLOGY}

The software of a system is similar to the human mind; it issues a command to control the hardware (the body) to perform a corresponding action. Hardware and software coordinate and actively cooperate to complete a task. System software design can be divided into transmission terminal and data center software designs.

The collector initially receives data through analog-todigital data conversion via the STM32 module to process data and then send them out. When the GPRS module is in a normal operation state, the link signal is sent through the SIM card to request a wireless communication link. The application layer HTTP will be the final storage after the data packet is judged by the three-handshake response.

The implementation of the program structure is determined according to the use of system hardware resources and the design of real-time requirements. The transmission terminal of the system mainly performs data receiving and sending through an interruption. After the transmission terminal is started, the corresponding module is initialized. Serial port 3 is opened to receive the data collected by the sensor to ensure the real-timeliness and integrity of the data. The interruption is then set as interruption priority. When the interface receives the data, the interruption is generated, and the data are received and stored. The sensor will be collected toward the data package sent to serial port 3 . Serial port 3 receives the data packets to determine the effectiveness of processing and extracts valid data through STM32 serial port 2 to send them out. The data are returned to close the serial port.

The main functions of the software system to implement the main program are as follows: system start-up, system initialization, calculation of the last accumulated locomotive time, saving to external data memory, GPRS wireless transmission module for sending data, receiving global positioning system (GPS) location and time information, real-time acquisition speed sensor square wave signal, and liquid crystal display system flowchart (Fig.1).

\section{A. Sensor Node Data Processing}

Sensor data fusion is a highly targeted technology that is widely used in industrial control, agricultural production, military, transportation, construction, and other fields. The 


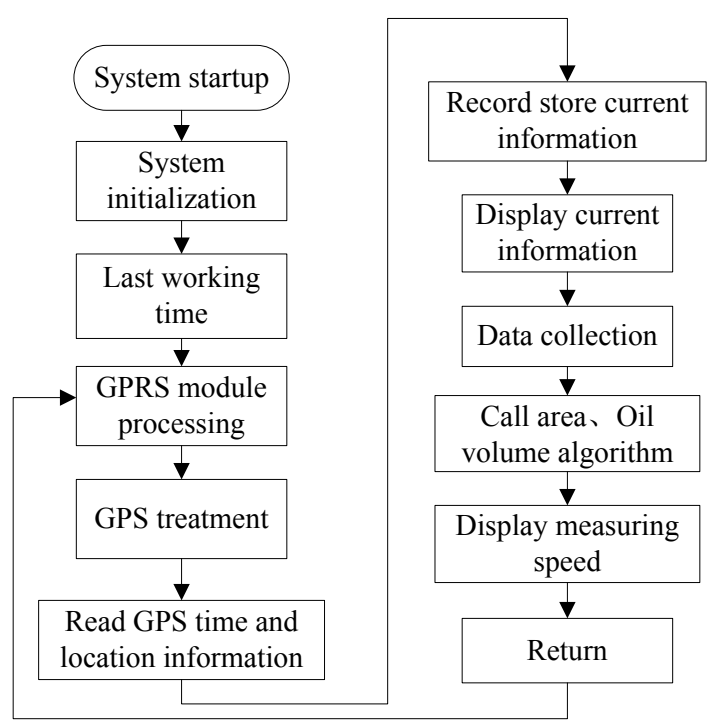

Figure 1. Flowchart of the main program

information provided by a variety of sensors is considerable; hence, we need a technology that can effectively deal with a large number of sensor data. Sensor data fusion is the procedure of using the information collected by multiple sensors to be processed in space and time or in accordance with the rules of a particular combination of complementary information to obtain a precise description of the object being monitored.

Common data fusion algorithms used in practical applications can be divided into two categories, namely, modern data fusion algorithms and classical data fusion algorithms. Classical data fusion algorithms based on classical mathematics can be divided into mathematical statistics methods and mathematical estimation methods according to the mathematical tools used, such as Bayes estimation, Kalman filter, and so on. With the development of modern information theory, artificial intelligence, and other disciplines, such as the neural network data fusion algorithm, conducting model building and calculation using classical algorithms have become difficult; high performance and general applicability are required.

Performing the reasoning of these data is necessary because of the uncertainty in the source information obtained from many nodes in a sensor network. The neural network data fusion algorithm requires that we first need to obtain the sensor network nodes of the weight distribution based on the similarity between the source data for classification and the source data for comparison. A specific neural network learning method is adopted to acquire training information and the corresponding data-reasoning mechanism.

In the sensor data fusion algorithm based on a neural network, the network has a unified form of information expression. The neural network learning method is adopted to achieve the fusion of source data and to determine related network parameters. Uncertain data can be converted into the system via reasoning and learning to address certainty in data information. Training rules can be expressed in data amount, and a training knowledge base is established on the basis of training rules. The fusion algorithm based on a neural network has considerable parallel data-processing capabilities; therefore, it can achieve rapid processing of system data.
ZigBee wireless network sensor nodes are utilized to improve the accuracy of data acquisition. Raw data are pretreated to eliminate errors. Node computation and data storage capabilities are considered. The pretreatment process collects data at the sensor nodes, which are a certain number of data after several cycles. The design process is as follows [5].

Nodes periodically collect data. When the last data are stored in the buffer queue, the queue is filled, and the node does not enter the dormant state instead of data preprocessing [6].

Nodes remove the sensor data in the buffer queue. The mean value is defined according to their values from small to large arrays as follows:

$$
\begin{array}{r}
V_{m}=\frac{V_{N}+1}{2}, \\
V_{m}=\frac{\frac{V_{N}+1}{2}+\frac{V_{N}}{2}}{2} .
\end{array}
$$

The computational dispersion presents

$$
S_{d}=V_{q}-V_{p}
$$

The invalid judgment interval for this group is

$$
\left|V_{i}-V_{m}\right|>\beta \times S_{d}(\beta=2) .
$$

The interval boundary of the data is obtained as

$$
\begin{aligned}
& \rho_{1}=V_{p}-\frac{\beta}{2} \times S_{d}, \\
& \rho_{1}=V_{q}-\frac{\beta}{2} \times S_{d} .
\end{aligned}
$$

Finally, the sensor nodes in the network will remove the measured data from the deviation value through the wireless channel to the embedded web server for further processing.

To collect information, wireless sensor networks rely on a large number of sensor nodes to cooperate with one another to collect and process the information of monitoring objects in the network coverage and to send it to the observation center. In the layout of wireless sensor nodes, a certain amount of data must be configured to solve redundant nodes, node measurement error, node failure, and other issues to satisfy application requirements. Determining information accuracy in the industrial control field is difficult. Similarly, identifying the comprehensive characteristics of complex objects is also difficult. Accordingly, the system should provide sufficient support in terms of the number and type of sensors. This condition will inevitably cause the sensor nodes to overlap with one another, which leads to data redundancy. The redundancy of the original data will provide wireless sensor network nodes with frequent access to channel competition, reduce the efficiency of data acquisition, and waste the limited energy of sensor nodes and communication bandwidth, thereby resulting in increased system delay. 
The sensor data fusion technology can effectively reduce redundant information, improve the efficiency of information collection and node energy use, and enhance the performance of the monitoring system.

In the ZigBee wireless sensor network, the radio frequency (RF) unit that completes a bit of data to send and receive node energy consumption is called the node microcontroller unit to execute an energy consumption command hundreds of times to as much as a thousand times. Therefore, the initial data are processed using the computing power of nodes in WSNs, which can reduce the frequency of the RF transceiver and the energy consumption of nodes.

\section{B. Verification of Data Fusion Design}

The effectiveness of the sensor data fusion design is validated. The sensor nodes are divided into two cluster structures, with each cluster containing five nodes. The test temperature values are shown in Figure 2.

Compared with the arithmetic mean method, the fusion method adopted in the design can effectively improve measurement accuracy $[7,8]$.

The design consists of sensor nodes and a web server two-level data fusion scheme to fulfill the requirements of the monitoring glass tempering furnace and to address the data redundancy caused by reducing the large capacity of the ZigBee WSN [9-11]. The system can effectively reduce communication amount, decrease the energy consumption of nodes [12-14], prolong network time, and provide accurate data measurement.

\section{RESUlt ANALYSIS AND DISCUSSION}

The entire network system research and design are described. The corresponding hardware or software is tested and regulated to ensure the normal operation of the system. The testing of the hardware and the development program regulation are explained.

The test table of a precision seeding machine for soybeans is shown in Figure 4.

The precision agricultural system is installed in the laboratory bench test. The concrete is in the soybean precision seeder test bed, with six rows of metering tubes. A photosensitive sensor and a photoelectric sensor are mounted on the conductor pipe. The photoelectric sensor is installed in the guide tube. When the seed is mediated through a tube and a seed interdiction sensor light source, the photoelectric tube emits a signal that indicates that the sowing machine is working normally. When a fault occurs in row sowing within the prescribed period, an alarm will be activated. The bench-seeding monitoring platform during a physical experiment is shown in Figure 5.

The specific operation is performed in a normal seeding planter with a six-row tube processed by a human. The alarm response time of the seeder-monitoring system is recorded. When a row is blocked, the node buzzer can send data to monitor the master node in the terminal display. The alarm response time of the controller area network (CAN) bus to transmit data has a time resolution of $0.1 \mathrm{~ms}$ while recording the row number. After several tests, two groups of repeated trials are selected. The data recorded are shown in Tables I and II.

During the specific operation, i.e., the process of the sowing machine, the alarm response time of the monitor-

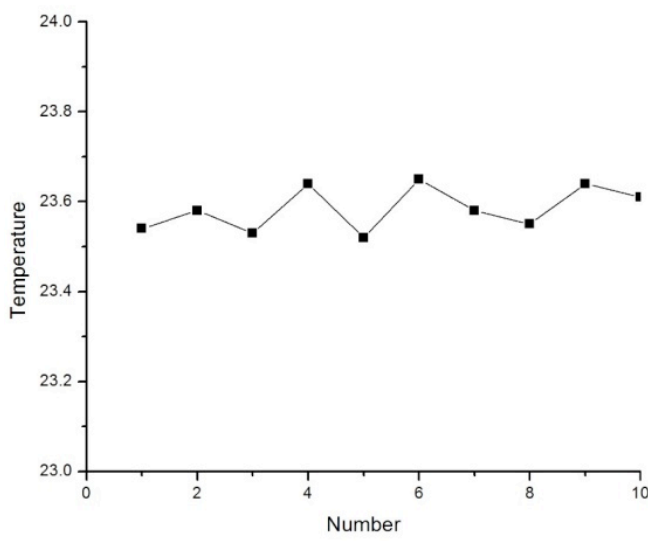

Figure 2. Measured temperature

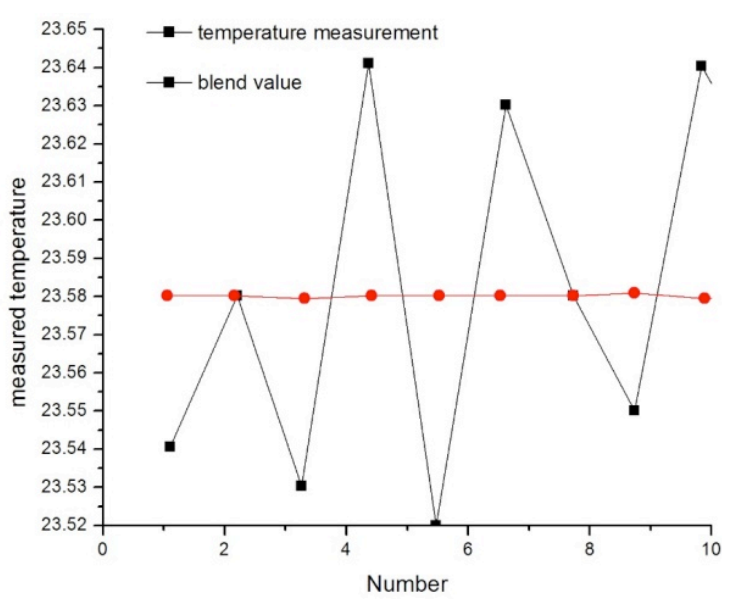

Figure 3. Comparison of the temperature values before and after data fusion

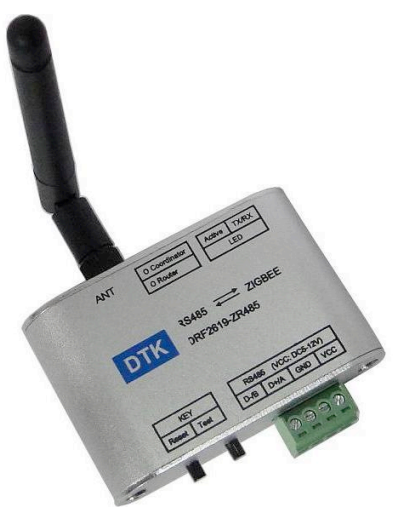

Figure 4. Test table of a precision seeding machine for soybeans

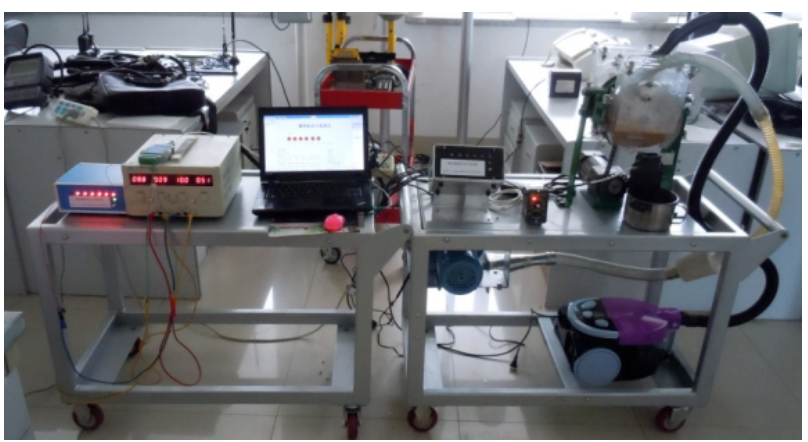

Figure 5. Bench-seeding monitoring platform 
TABLE I. PERFORMANCE TEST DATA (1)

\begin{tabular}{|l|c|c|c|c|c|c|}
\hline $\begin{array}{l}\text { Main monitoring node record } \\
\text { number }\end{array}$ & 1 & 2 & 3 & 4 & 5 & 6 \\
\hline Response time (ms) & 28.5 & 29.2 & 27.3 & 35.4 & 29.3 & 36.8 \\
\hline Communication is correct & No & No & No & No & No & No \\
\hline
\end{tabular}

TABLE II. PERFORMANCE TEST DATA (2)

\begin{tabular}{|l|c|c|c|c|c|c|}
\hline $\begin{array}{l}\text { Main monitoring node record } \\
\text { number }\end{array}$ & 1 & 2 & 3 & 4 & 5 & 6 \\
\hline Response time (ms) & 38.5 & 28.2 & 35.7 & 35.4 & 39.2 & 36.3 \\
\hline Communication is correct & No & No & No & No & No & No \\
\hline
\end{tabular}

ing system of the seeding machine is recorded by a person and is blocked by the six channels. When a row of pipe is blocked, the buzzer will be activated. CAN then sends data to monitor the main node in the terminal display alarm barrier, i.e., CAN alarm response time. The time resolution of the CAN bus data transmission is $0.1 \mathrm{~ms}$. The main section of the 40 points records and monitors the seed tube. After numerous tests, three groups are selected to repeat the test.

The sowing sensor sends a message to the PC. The staff can then monitor the master station and solve a problem according to a specific set of message protocols by reading the frame start symbol, ID, and seeding information. The data frame in the seeded number can be evaluated using cyclic query and the judgment analysis method. The specific information that corresponds to the seed tube row number is displayed. Consequently, the staff can monitor the actual situation of sowing at a glance and prevent leakage, thereby reducing agricultural losses.

The seeding-monitoring system can continuously work for $24 \mathrm{~h}$ or $48 \mathrm{~h}$, and the hardware and software designs of the system are relatively stable.

Through numerous experiments, this study determines that the monitored and actual situations coincide without errors. The response time is short, which is in accordance with the sowing requirements. The system design is also reasonable and fulfills the requirements.

\section{CONCLUSIONS}

With the continuous progress of science and technology, IT is increasingly applied to traditional agriculture. IT is used in agricultural production to improve the efficiency of agricultural planting, increase the input-output ratio of agricultural production, acquire high-quality agricultural products, and reduce the amount of labor in the process of agricultural production. A remote monitoring and control system for a sowing machine with wireless information transmission function is constructed based on ARM embedded technology. This system can effectively improve agricultural planting efficiency and sowing quality, considerably reduce the workload of farmers, and enhance land utilization. The system uses the ARM core microcontroller for control, and the hardware design is realized using an embedded technology and the ZigBee module. Hence, this system can monitor field work in real time. Locomotive information can be transmitted to the data center through wireless transmission technology, and data can be regarded in real time. This system can rapidly monitor the agricultural seeding machine while it is operating. Overall, the proposed system can effectively promote the rapid development of modern agriculture.

\section{REFERENCES}

[1] Safari, Hanieh, Thomas Fricke, and Michael Wachendorf. "Determination of fibre and protein content in heterogeneous pastures using field spectroscopy and ultrasonic sward height measurements." Computers and Electronics in Agriculture, 2016, vol. 123, pp. 256-263. http://dx.doi.org/10.1016/j.compag.2016.03.002

[2] Agarwal, Aashish. "Color Tuneable Bio-Sensor Made Up From Stimuli-Responsive Nanoparticles," International Journal of Students' Research in Technology \& Management," 2016, vol. 4, no.1, pp. 06-07.

[3] Luvisi, Andrea, Alessandra Panattoni, and Alberto Materazzi, "RFID temperature sensors for monitoring soil solarization with biodegradable films," Computers and Electronics in Agriculture, 2016, vol. 123, pp. 135-141. http://dx.doi.org/10.1016/j.compag. 2016.02 .023

[4] Bourgeon, Marie-Aure, et al., "Field radiometric calibration of a multispectral on-the-go sensor dedicated to the characterization of vineyard foliage," Computers and Electronics in Agriculture, 2016 vol. 123, pp. 184-194. http://dx.doi.org/10.1016/j.compag.2016. $\underline{02.019}$

[5] Bilsborrow, R. E., "Population pressures and agricultural development in developing countries: A conceptual framework and recent evidence," World Development, 1987, vol.15, no.2, pp. 183203. http://dx.doi.org/10.1016/0305-750X(87)90077-5

[6] Roy, Indrajit, et al. "Effect of Supplemental Application of Nitrogen, Irrigation and Hormone on the Yield and Yield Components of Chickpea," World Journal of Agricultural Sciences, 2016, vol. 12.1, pp. 70-77.

[7] Davis, A. M., Tink, M., Rohde, K., \& Brodie, J. E. "Urea contributions to dissolved 'organic'nitrogen losses from intensive, fertilised agriculture," Agriculture, Ecosystems \& Environment, 2016 , vol. 223, pp. 190-196. http://dx.doi.org/10.1016/j.agee.2016. 03.006

[8] Li, J., Zhu, T., Mao, X., \& Adeloye, A. J., "Modeling crop water consumption and water productivity in the middle reaches of Heihe River Basin," Computers and Electronics in Agriculture, 2016, vol. 123, pp. 242-255. http://dx.doi.org/10.1016/j.compag.2016. $\underline{02.021}$

[9] Alhmiedat T. "An Adaptive Energy-Efficient Data Collection System for ZigBee Wireless Sensor Networks". International Journal of Distributed Sensor Networks, 2015. http://dx.doi.org/10.1155/2015/734937

[10] Khan M, Passerone R, Macii D. "FZepel: RF-level power consumption measurement (RF-PM) for Zigbee wireless sensor network-towards cross layer optimization". Emerging Technologies and Factory Automation, 2008.

[11] Boudhir A, Mohamed B. "New Technique of Wireless Sensor Networks Localization based on Energy Consumption". International Journal of Computer Applications, 2010, vol. 9, no. 12, pp. 24-28. http://dx.doi.org/10.5120/1436-1935

[12] Kong J, Kim J, Eom D. "Energy-Aware Distributed Clustering Algorithm for Improving Network Performance in WSNs". International Journal of Distributed Sensor Networks, 2014. http://dx.doi.org/10.1155/2014/670962

[13] Babaie S, Zade A, Hosseinalipour A. "New clustering method to decrease probability of failure nodes and increasing the lifetime in WSNs". Clinical Orthopaedics and Related Research, 2010.

[14] Lin K, Chen M, Zeadally S. "Balancing energy consumption with mobile agents in wireless sensor networks". Future Generation Computer Systems, 2012, vol. 28, no. 2, pp.446-456. http://dx.doi.org/10.1016/j.future.2011.03.001

\section{AUTHORS}

Jiaqi He is with the Department of Eco-agriculture and Rural Development, Sichuan Agricultural University, Sichuan, China (e-mail: 195473616@qq.com).

Yong Chen (corresponding author) is with the Department of Eco-agriculture and Rural Development, Sichuan Agricultural University, Sichuan, China (e-mail: yc-yong-chen@163.com).

Submitted, January, 20, 2016. Published as resubmitted by the authors on May, 142016. 